\title{
Structured symmetric rank-one method for unconstrained optimization.
}

\begin{abstract}
In this paper, we investigate a symmetric rank-one (SR1) quasi-Newton (QN) formula in which the Hessian of the objective function has some special structure. Instead of approximating the whole Hessian via the SR1 formula, we consider an approach which only approximates part of the Hessian matrix that is not easily acquired. Although the SR1 update possesses desirable features, it is unstable in the sense that, it may not retain positive definiteness and may become undefined. Therefore, we describe some safeguards to overcome these difficulties. Since the structured SR1 method provides a more accurate Hessian approximation, therefore the proposed method reduces significantly the computational efforts needed in solving a problem. The results of a series of experiments on a typical set of standard unconstrained optimization problems are reported, which show that the structured SR1 method exhibits a clear improvement in numerical performance over some existing QN algorithms.
\end{abstract}

Keyword: Structured quasi-Newton method; Unconstrained optimization; Symmetric rankone update; Hessian approximation; Partial information on the Hessian. 